\title{
Polish regional differences in patient knowledge on atrial fibrillation and its management as well as in patterns of oral anticoagulant prescription
}

\author{
Agnieszka Janion-Sadowska1, Marcin Sadowski¹, Małgorzata Konieczyńska², Grzegorz Skonieczny³, \\ Agnieszka Metzgier-Gumiela3 ${ }^{3}$, Magdalena Chrapek ${ }^{4}$, Ewa Sobieraj2,5, Agata H. Bryk ${ }^{2,5}$, Maciej Dębski ${ }^{2,5}$, \\ Piotr Podolec 2,5 , Barbara Małecka2,5, Lien Desteghe ${ }^{6,7,8}$, Hein Heidbuchel6,8, Anetta Undas 2,5 \\ 1 Faculty of Medicine and Health Sciences, Jan Kochanowski University, Kielce, Poland \\ 2 John Paul II Hospital, Kraków, Poland \\ 3 Provincial Polyclinical Hospital, Toruń, Poland \\ 4 Institute of Mathematics, Group of the Probability Calculus and Statistic, Jan Kochanowski University, Kielce, Poland \\ 5 Institute of Cardiology, Jagiellonian University Medical College, Kraków, Poland \\ 6 Faculty of Medicine and Life Sciences, Hasselt University, Hasselt, Belgium \\ 7 Heart Center Hasselt, Jessa Hospital, Hasselt, Belgium \\ 8 University of Antwerp and Antwerp University Hospital, Antwerp, Belgium
}

\section{KEY WORDS}

atrial fibrillation, knowledge, non-vitamin $\mathrm{K}$ antagonist oral anticoagulants, questionnaire, vitamin K antagonist

\section{EDITORIAL}

page 415
Correspondence to: Agnieszka Janion-Sadowska, MD, PhD, Faculty of Medicine and Health Sciences, Jan Kochanowski University, ul. Grunwaldzka 45, 25-736 Kielce, Poland, phone: +48413496970, email: ajanion@02.pl Received: January 24, 2019 Revision accepted: February 26, 2019 Published online: February 26, 2019 Kardiol Pol. 2019; 77 (4): 437-444 doi:10.5603/KP.a2019.0036 Copyright by Polskie Towarzystwo Kardiologiczne, Warszawa 2019

\section{ABSTRACT}

BACKGROUND The Jessa Atrial Fibrillation Knowledge Questionnaire (JAKQ) was successfully used to assess knowledge gaps in patients with atrial fibrillation (AF).

AIMS To evaluate the regional differences among Polish patients in their awareness of AF diagnosis and oral anticoagulation use.

METHODS A total of 1583 patients with AF at a median (IQR) age of 72 (66-79) years completed the JAKQ in 3 cardiology centers (center I, Kraków; center II, Toruń; center III, Kielce) from January 2017 to June 2018. The final analysis included 1525 patients, 32.9\% were on vitamin $\mathrm{K}$ antagonists (VKAs) and 67.1\% on non-VKA oral anticoagulants (NOACS), that is, rivaroxaban and dabigatran ( $28.9 \%$ each), and apixaban (9.3\%).

RESULTS The mean (SD) score on the JAKQ was $55.5 \%$ (18.4\%) with better results among patients on VKAs compared with NOACs (58\% [18.3\%] vs 54.3\% [18.4\%]; $P=0.0002)$ with time from AF diagnosis more than 12 months (57.4\% [17.5\%] vs 50\% [19.9\%]; $P<0.0001)$. There was a significant difference in the knowledge scores between the 3 centers (I, 59.5\%; II, 48.5\%; III, 54.3\%; $P<0.0001$ ). In all centers the number of correct answers correlated inversely with patient's age $(r=-0.20 ; P<0.0001)$. NOACs were more frequently used in center III. The percentage of correct responses was lower in patients on reduced NOAC doses (35.4\% of patients on NOACs), compared with the full-dose NOAC groups in center I $(56.9 \%$ vs $62.5 \% ; P=0.012)$ and II ( $48.1 \%$ vs $56.2 \% ; P=0.003)$.

CONCLUSIONS Patients from a high-volume academic center showed better knowledge than their peers from district hospitals. There are large regional differences in prescription patterns of oral anticoagulants, including the preferred NOAC.

INTRODUCTION Atrial fibrillation (AF) is a chronic condition that requires lifelong anticoagulation in most patients. Oral anticoagulants (OACs), both vitamin K antagonists (VKAs) and non-VKA oral anticoagulants (NOACs), diminish the risk of stroke in patients with $\mathrm{AF}$, but also increase the rate of bleeding. ${ }^{1}$ To use OAC most effectively at the lowest risk, patients must be fully compliant. The attending physicians who diagnose AF and initiate OAC therapy serve as a 


\section{WHAT'S NEW?}

Considerable differences in the prescription patterns of oral anticoagulants, including vitamin $\mathrm{K}$ antagonists and non-vitamin $\mathrm{K}$ antagonist oral anticoagulants, and in knowledge of atrial fibrillation were observed in large cardiology departments of 3 Polish cities, Kraków, Toruń, and Kielce. The Jessa Atrial Fibrillation Knowledge Questionnaire was used for the assessment. These differences may impact the effectiveness and safety of oral anticoagulant therapy and may help to set regional educational aims for specific atrial fibrillation populations. Further efforts are needed to improve knowledge about atrial fibrillation and its anticoagulant therapy in Poland and in other countries. center with 3 university departments, 1 cardiology ward (105544 hospitalized patients in 2017), and 36742 outpatients. Center II (Cardiology Ward, District Hospital in Toruń) had 3200 inpatients and 5650 outpatients in 2017. Center III (the Świętokrzyskie Cardiology Centre in Kielce) had 7602 inpatients and 9278 outpatients in 2017.

Patients participating in the study were older than age 18 years and were able to provide consent. The questionnaire was completed in the presence of a physician.

The questionnaire The JAKQ was used after obtaining formal consent from Hasselt University, Belgium. The questionnaire consists of 16 multiple choice questions with 1 correct answer, which was previously described in detail by Konieczyńska et al. ${ }^{8}$ The detailed summary is available in the Supplementary material. The final analysis included in this article conformed to the original JAKQ analysis by Desteghe et $\mathrm{al}^{4}$ and to the analysis by Konieczyńska et al. ${ }^{8}$ If a patient provided 2 or more responses despite clear instructions, the response was assessed as incorrect. Doctors who collected the questionnaires completed clinical data about OAC, its dose, time of treatment, time of diagnosis, comorbidities, antiplatelet use, and prior severe bleeding or cerebrovascular ischemic events. Comorbidities were defined as presented in Supplementary material. Bleeding severity was defined according to the International Society of Thrombosis and Haemostasis. ${ }^{9}$

Statistical analysis The total score was presented as the percentage of correct answers. Categorical data were presented as numbers and percentages. The $\mathrm{X}^{2}$ test or Fisher exact test were used to compare proportions. Numerical variables were presented as mean and SD or median and interquartile range (IQR), as appropriate, and compared by the $t$ test, 1-way analysis of variance (ANOVA), Mann-Whitney test, or Kruskal-Wallis test, as appropriate. The relationship between the number of correct answers and age as well as between time since AF onset and time since $\mathrm{OAC}$ initiation was assessed by the Spearman rank correlation coefficient. A $P$ value below 0.05 was considered statistically significant. All statistical analyses were performed using the R statistical software (version 3.1.2; The R Foundation for Statistical Computing, Vienna, Austria).

RESULTS Patient characteristics A total of 1583 patients completed the questionnaire. The answers of 28 individuals (1.8\%) were excluded because low-molecular-weight heparin was used as an anticoagulant, and 30 (1.9\%) because data were missing. The final analysis encompassed 
1525 patients (96.3\%), including 585 outpatients (38.4\%) and 940 hospitalized patients (61.6\%). Age of the patients ranged from 22 to 96 years (median [IQR], 72 [66-79]). Women represented $45.5 \%$ of the study population. Paroxysmal and permanent $\mathrm{AF}$ were the most common types of the disease $(43.7 \%$ and $39.5 \%$, respectively). The median (IQR) time from AF diagnosis was 48 (12-108) months and median (IQR) anticoagulation duration was 18 (5-48) months. The majority of the patients (67.1\%) were treated with NOACs, that is, rivaroxaban and dabigatran (28.9\% each), and apixaban (9.3\%), and one-third received warfarin or acenocoumarol (32.9\%). Concomitant antiplatelet therapy was identified in $22.3 \%$ of patients (aspirin, $15 \%$ and clopidogrel, 7.3\%).

Comparison of the 3 centers The youngest patients came from center I, and the oldest from center II (TABLE1). Center II had more patients with persistent $\mathrm{AF}$, a shorter time since the onset of $\mathrm{AF}$, and the shortest anticoagulation duration compared with the other centers. NOAC use was reported more frequently in center III. Rivaroxaban was administered more often in center II (48.1\%), and dabigatran in center III (54.6\%). Center I prescribed rivaroxaban (47.1\%) and dabigatran (41.8\%) at similar rates. Among patients treated with NOAC at reduced doses, $15 \mathrm{mg} / \mathrm{d}$ of rivaroxaban was most commonly used in centers I and II. Patients from center I suffered more commonly from arterial hypertension, diabetes, and vascular disease. Prior stroke or transient ischemic attack was observed more often in centers I and III, while patients with prior myocardial infarction were overrepresented in center II (TABLE1).

The mean (SD) score on the JAKQ was 55.5\% (18.4\%) with no significant difference between men and women $(54.8 \%$ [18.1\%] and 56.3\% [18.8\%], respectively; $P=0.13$ ). Better results were obtained in patients under the age of 75 years as compared with those older than 75 years of age (58.1\% [17.2\%] and 50.8\% [19.7\%], respectively; $P<0.0001)$, treated with VKAs as compared with those treated with NOACs (58\% [18.3\%] and 54.3\% [18.4\%], respectively; $P=0.0002)$, with more than 12 months since the AF diagnosis as compared with those with less than 12 months (57.4\% [17.5\%] and 50\% [19.9\%], respectively; $P<0.0001$ ), with anticoagulation duration over 1 year as compared with those with anticoagulation duration of less than 1 year (58.3\% [17.6\%] and 52.1\% [18.9\%], respectively; $P<0.0001$ ). Patients receiving clopidogrel as compared with those not receiving this drug (48.9\% [19\%] and 56\% [18.3\%], respectively; $P=$ 0.0002 ) had results almost identical to those diagnosed with vascular disease as compared with those without vascular disease (52\% [19\%] and $56.5 \%$ [18.2\%], respectively, $P<0.0001)$. Lower scores were achieved by patients with prior myocardial infarction as compared with those without prior myocardial infarction $(52.7 \%$ [18.2\%] and $56.3 \%[18.2 \%]$ respectively; $P=0.001)$. The same was true for diabetic patients as compared with those without this disease (54\% [19\%] and 56.\% [18.1\%], respectively; $P=0.033$ ), and patients with heart failure as compared with those without heart failure $(54.2 \%$ [19.5\%] and $56.5 \%$ [18.3\%], respectively; $P=0.019$ ), with a similar prevalence of these diseases in most patients. Three questions with the highest percentage of correct responses were those regarding the indication for OAC (84.4\%), related to the recommended frequency of measurement of international normalized ratio (90.6\%), and concerning the importance of fixed hours of taking NOAC (90.4\%). Poor patient knowledge was observed in the topics of the effectiveness of antiarrhythmic drugs (31\% of correct answers) and procedures in case of anticoagulant dose omission (VKA group, 24.1\%; NOAC group, 38.4\%).

The worst scores were achieved by patients from center II (I, 59.5\%; II, 48.5\%; III, 54.3\%; $P<0.0001$; FIGURE 1). The following topics were associated with a similar number of correct responses in all 3 centers: the importance of a regular anticoagulant intake despite the lack of symptoms, frequency of international normalized ratio measurements, and taking NOAC at fixed hours. Patients from center III were more aware that overweight exacerbates AF (I, 53.1\%; II 53.6\%; III, $62.4 \% ; P=0.003$ ) and more familar with the procedure in case of omitting a dose of VKA (I, 21.4\%; II, 17.8\%; III, 33.6\%; $P=0.007$ ). Patients from center II had the lowest number of correct answers regarding lack of symptoms in AF (I, 35.0\%; II, 22.1\%; III, 39.8\%; $P<0.0001$ ) and the need to consult their physician before planned surgery while on OAC (I, 72.5\%; II, 65.7\%; III, 74.7\%; $P=0.019$ ).

Weak positive correlations were observed between the number of correct answers and the time from AF onset $(r=0.13 ; P<0.0002)$ and from OAC initiation $(r=0.18 ; P<0.0003)$. There was an inverse association between the number of correct answers and the patient's age $(r=-0.20 ; P<0.0001)$ in the whole group. Similar observations were made for participating centers; however, there was no correlation between the knowledge score and the time from AF onset in center I (I, $r=0.05 ; P=0,18$; II, $r=0.14$; $P$ $=0.009$; III, $r=0.19 ; P=0.000017$ ).

Knowledge on vitamin $K$ antagonists compared with non-vitamin $\mathrm{K}$ antagonist oral anticoagulants In the whole study group, patients on NOACs more often had paroxysmal AF and shorter time from AF diagnosis and OAC initiation. They less frequently had heart failure, diabetes, and mitral stenosis. The incidence of antiplatelet drug use and major bleedings was 
TABLE 1 Patient characteristics

\begin{tabular}{|c|c|c|c|c|}
\hline Variable & $\begin{array}{l}\text { Center I } \\
\quad(n=702)\end{array}$ & $\begin{array}{l}\text { Center II } \\
\quad(n=321)\end{array}$ & $\begin{array}{r}\text { Center III } \\
(n=502)\end{array}$ & $P$ value \\
\hline \multicolumn{5}{|l|}{ Demographic data } \\
\hline Age, y, median (IQR) & $70(64-78)$ & $74(66-80)$ & $72(65-79)$ & 0.0003 \\
\hline Female sex & $327(46.6)$ & $132(41.1)$ & $233(46.4)$ & 0.23 \\
\hline \multicolumn{5}{|l|}{ AF type } \\
\hline Paroxysmal & $314(44.7)$ & $121(37.7)$ & $232(46.2)$ & \multirow[t]{4}{*}{$<0.0001$} \\
\hline Persistent & $111(15.8)$ & $94(29.3)$ & $44(8.8)$ & \\
\hline Permanent & $271(38.6)$ & $106(33)$ & $226(45)$ & \\
\hline Unknown & $6(0.9)$ & $0(0)$ & $0(0)$ & \\
\hline Time since AF onset, mo, median (IQR) & $48(18-96)$ & $30(7-96)$ & $48(12-120)$ & 0.0004 \\
\hline Time since OAC initiation, mo, median (IQR) & $22(8-48)$ & $8(2-28)$ & $18(4-47.5)$ & $<0.0001$ \\
\hline \multicolumn{5}{|l|}{ OAC type } \\
\hline NOAC & $450(64.1)$ & $214(66.7)$ & $359(71.5)$ & 0.025 \\
\hline Rivaroxaban 20 mg once daily & $145(20.7)$ & 64 (19.9) & $90(17.9)$ & 0.49 \\
\hline Rivaroxaban 15 mg once daily & $70(10)$ & $39(12.1)$ & $33(6.6)$ & 0.017 \\
\hline Dabigatran 150 mg twice daily & $125(17.8)$ & $33(10.3)$ & $125(24.9)$ & $<0.0001$ \\
\hline Dabigatran 110 mg twice daily & $63(9)$ & $23(7.2)$ & $71(14.1)$ & 0.002 \\
\hline Apixaban 5 mg twice daily & $21(3)$ & $37(11.5)$ & $21(4.2)$ & $<0.0001$ \\
\hline Apixaban 2.5 mg twice daily & $26(3.7)$ & $18(5.6)$ & $19(3.8)$ & 0.34 \\
\hline VKA & $252(35.9)$ & $107(33.3)$ & $143(28.5)$ & 0.025 \\
\hline Acenocoumarol & $133(18.9)$ & $53(16.5)$ & $89(17.7)$ & 0.64 \\
\hline Warfarin & $119(17)$ & $54(16.8)$ & $54(10.8)$ & 0.005 \\
\hline \multicolumn{5}{|l|}{ Antiplatelet therapy } \\
\hline Aspirin & $123(17.5)$ & $66(20.6)$ & $39(7.8)$ & $<0.0001$ \\
\hline Clopidogrel & $32(4.6)$ & $58(18.1)$ & $21(4.2)$ & $<0.0001$ \\
\hline \multicolumn{5}{|l|}{ Comorbidities } \\
\hline Heart failure & $283(40.3)$ & $143(44.5)$ & $226(45)$ & 0.20 \\
\hline Arterial hypertension & $601(85.6)$ & $257(80.1)$ & $399(79.5)$ & 0.009 \\
\hline Diabetes & $250(35.6)$ & $95(29.6)$ & $141(28.1)$ & 0.014 \\
\hline Prosthetic valve & $53(7.5)$ & $20(6.2)$ & $44(8.8)$ & 0.43 \\
\hline Mitral stenosis & $23(3.3)$ & $7(2.2)$ & $15(3)$ & 0.68 \\
\hline Prior myocardial infarction & $151(21.5)$ & $99(30.8)$ & $113(22.5)$ & 0.004 \\
\hline Vascular disease & $215(30.6)$ & $36(11.2)$ & 85 (16.9) & $<0.0001$ \\
\hline Prior major bleeding & $80(11.4)$ & $19(5.9)$ & $48(9.6)$ & 0.019 \\
\hline VKA in the past & $143(20.4)$ & $62(19.3)$ & $112(22.3)$ & 0.56 \\
\hline
\end{tabular}

Data are presented as numbers and percentages unless otherwise stated.

Abbreviations: AF, atrial fibrillation; IQR, interquartile range; NOAC, non-vitamin K antagonist oral anticoagulant; OAC, oral anticoagulant; VKA, vitamin K antagonist 
FIGURE 1

Distribution of correct responses in patients treated with non-vitamin $\mathrm{K}$ antagonist oral anticoagulants and vitamin Kantagonists

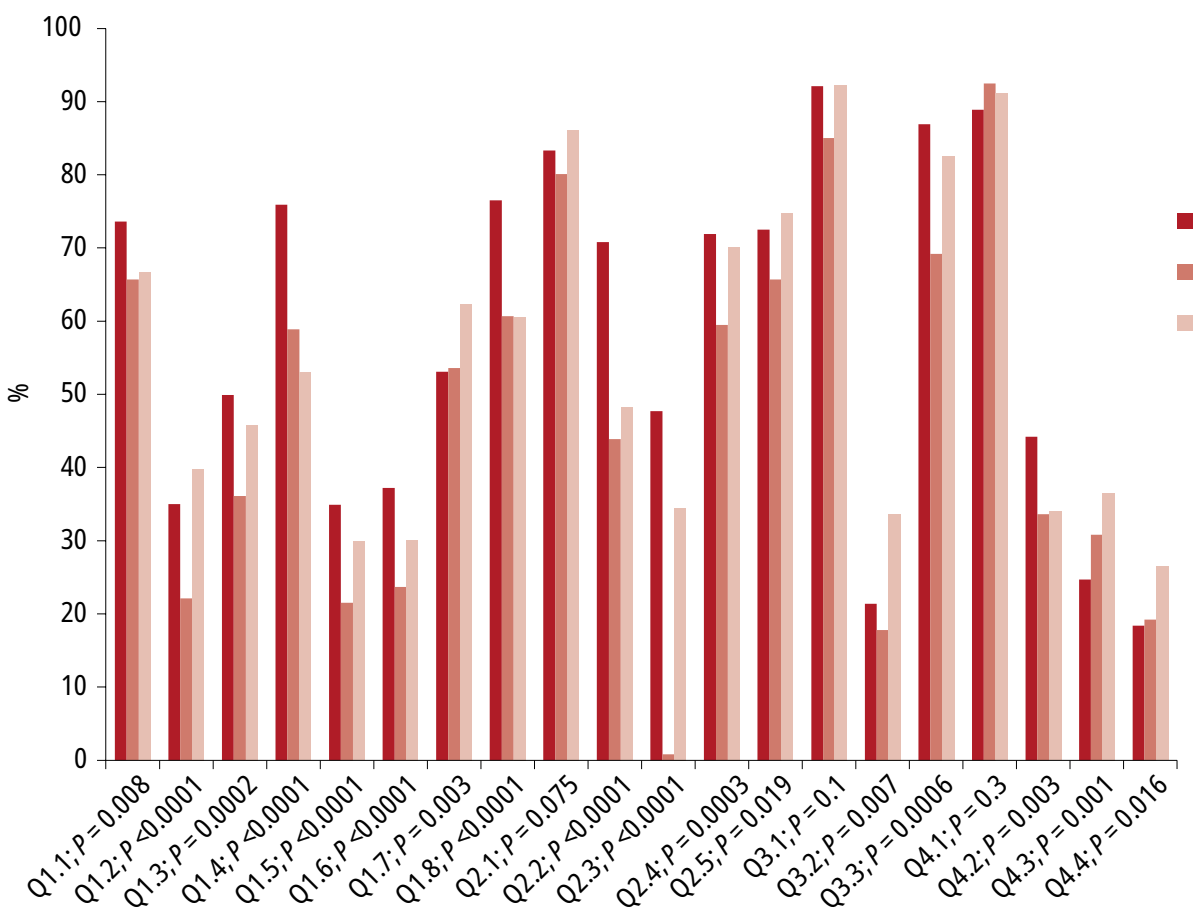

TABLE 2 Comparison of patients with atrial fibrillation treated with non-vitamin $\mathrm{K}$ antagonist oral anticoagulants and vitamin $\mathrm{K}$ antagonists

\begin{tabular}{|c|c|c|c|}
\hline Variable & NOAC $(n=1023)$ & VKA $(n=502)$ & $P$ value \\
\hline \multicolumn{4}{|l|}{ Demographic data } \\
\hline Age, y, median (IQR) & $71(64-79)$ & $72(66-79)$ & 0.18 \\
\hline Female sex & $481(47)$ & $211(42)$ & 0.07 \\
\hline \multicolumn{4}{|l|}{ AF type } \\
\hline Paroxysmal & $503(49.2)$ & $164(32.7)$ & \multirow[t]{4}{*}{$<0.0001$} \\
\hline Persistent & $215(21.0)$ & $34(6.8)$ & \\
\hline Permanent & $302(29.5)$ & $301(60.0)$ & \\
\hline Unknown & $3(0.3)$ & $3(0.6)$ & \\
\hline \multicolumn{4}{|l|}{ History of AF and anticoagulation } \\
\hline Time since AF onset, mo, median (IQR) & $27(10-60)$ & $96(40-156)$ & $<0.0001$ \\
\hline Time since OAC initiation, mo, median (IQR) & $11(3-24)$ & $60.0(24-120)$ & $<0.0001$ \\
\hline \multicolumn{4}{|l|}{ Antiplatelet therapy } \\
\hline Aspirin & $157(15.3)$ & $71(14.1)$ & 0.59 \\
\hline Clopidogrel & $81(7.9)$ & $30(6.0)$ & 0.21 \\
\hline \multicolumn{4}{|l|}{ Comorbidities } \\
\hline Heart failure & $378(37.0)$ & $274(54.6)$ & $<0.0001$ \\
\hline Arterial hypertension & $841(82.2)$ & $416(82.9)$ & 0.77 \\
\hline Diabetes & $303(29.6)$ & $183(36.5)$ & 0.008 \\
\hline Prosthetic valve & $8(0.8)$ & $109(21.7)$ & $<0.0001$ \\
\hline Mitral stenosis & $16(1.6)$ & $29(5.8)$ & $<0.0001$ \\
\hline Prior myocardial infarction & $233(22.8)$ & $130(25.9)$ & 0.18 \\
\hline Vascular disease & $206(20.1)$ & $130(25.9)$ & 0.012 \\
\hline VKA in the past & $317(31.0)$ & - & - \\
\hline
\end{tabular}

Data are presented as numbers and percentages unless otherwise stated. 
similar in both groups (TABLE 2). Those differences were consistent among all 3 centers (Supplementary Material, Table S1).

Patients on NOACs were more aware of AF complications compared with those on VKAs (67.4\% and 59.4\%, respectively; $P=0.002$ ). $\mathrm{Pa}-$ tients on VKAs, as compared with those on NO$\mathrm{ACs}$, achieved better scores in questions on the management in case of an AF episode $(38.4 \%$ and $28.8 \%$, respectively; $P=0.002$ ) and in questions regarding the indication for OAC $(86.9 \%$ and $81.9 \%$, respectively; $P=0.015$ ). There were no differences in distribution of correct answers to the other questions.

In center I, patients on NOACs, compared with those on VKAs, had better knowledge of AF selfdetection (52.9\% and $44.4 \%$, respectively; $P=$ $0.034)$ and possible complications $(80.9 \%$ and $67.1 \%$, respectively; $P<0.0001)$. However, patients receiving VKAs, as compared with those receiving NOACs, had a better awareness of the adequate self-management in case of an AF episode ( $87.3 \%$ and $81.1 \%$, respectively; $P=0.035$ ). There were no significant differences between both treatment groups in center II. Patients receiving VKAs, as compared with those receiving NOACs, in center III were more aware that $\mathrm{AF}$ is not always accompanied by symptoms $(49.7 \%$ and $35.9 \%$ respectively; $P<0.0001)$, were more familiar with side effects of anticoagulation $(55.9 \%$ and $45.1 \% ; P=0.03)$, and the management of minor bleedings $(77.6 \%$ and $67.1 \% ; P=0.023)$.

Patients on different non-vitamin $\mathrm{K}$ antagonist oral anticoagulants In the whole group, the proportion of patients on rivaroxaban and dabigatran were similar $(43.1 \%$ and $43.0 \%$, respectively), and the apixaban group was much smaller (13.9\%). Patients taking apixaban, as compared with those on rivaroxaban and dabigatran, were the oldest (median [IQR], I, 76 [68-81]; II, 71 [64-78]; III, 71 [63-78] years; $P=0.0002)$ and received anticoagulant therapy for the shortest time (I, 4 [1-10]; II, 14 [6-27]; III, 11 [3-24] months; $P<0.0001)$. Patients on apixaban more often received concomitant antiplatelet treatment (aspirin, I, 26.1\%; II, 13.4\%; III, $13.9 \%$; $P=0.001$; clopidogrel, I, 20.4\%; II, 6.1\%; III, 5.7\%; $P<0.0001)$. More patients on apixaban had myocardial infarction in the past (I, 38\%; II, 19.3\%; III, 21.4\%; $P<0.0001)$ in comparison with the rivaroxaban and dabigatran groups. $\mathrm{Pa}-$ tients on rivaroxaban, compared with those on dabigatran and apixaban, had the longest time from AF diagnosis (I, 36; II, 24; III, 22 months; $P=0.002)$.

In center I, more women (I, 61.7\%; II, 52.6\%; III, 43.1\%; $P=0.035$ ) and patients with prior stroke (I, 21.3\%; II, 9.8\%; III, $16.5 \%$; $P=0.038$ ) received apixaban than rivaroxaban or dabigatran.

In center II, age was similar in the 3 groups of patients receiving different NOACs. Patients on apixaban, as compared with patients on rivaroxaban and dabigatran, received antiplatelet agents more frequently (aspirin, 38.2\%, 13.6\%, and $17.9 \%$, respectively; $P=0.002$; clopidogrel, $40.4 \%, 14.3 \%$, and $8.7 \%$, respectively; $P<0.0001$ ).

There was no age difference in center III among patients taking different NOACs. $\mathrm{Pa}-$ tients treated with apixaban, compared with those on rivaroxaban and dabigatran, more often received aspirin $(20 \%, 6.5 \%$, and $8.7 \%$, respectively; $P=0.04$ ) and had prior myocardial infarction (50\%, $14.6 \%$, 21\%, respectively; $P<0.0001)$.

The proportion of correct responses in the JAKQ was similar in all NOAC groups; however, patients treated with rivaroxaban and dabigatran, as compared with those on apixaban, were more aware of the necessity of taking their drugs irrespectively of AF symptoms $(80.3 \%, 85.7 \%$, and $75.4 \%$, respectively; $P=0.01$ ).

Reduced NOAC doses were prescribed to $35.4 \%$ of the patients. Rivaroxaban was used in $32.2 \%$ of the patients, dabigatran in $35.7 \%$, and apixaban in $47.7 \%$. Patients receiving reduced NOAC doses, as compared with those receiving full doses, were older (80.0 [74.0-84.0] and 68 [61.0-73.0] years, respectively; $P<0.0001)$, were more often women $(52.8 \%$ and $43.9 \%$, respectively; $P=0.007$ ), more frequently used concomitant antiplatelet drugs (aspirin, 21.3\% and $12.1 \%$, respectively; $P=0.0001$; clopidogrel, $15.7 \%$ and $3.6 \%$, respectively; $P<0.0001$ ), and more often had history of heart failure $(45.9 \%$ and $32.1 \%$, respectively; $P<0.0001$ ), arterial hypertension $(85.6 \%$ and $80.3 \%$, respectively; $P$ $=0.04)$, and prior myocardial infarction $(33.4 \%$ and $16.9 \%$, respectively; $P<0.0001$ ). Patients on reduced NOAC doses had less correct responses as compared with those on full doses (mean [SD], $51.5 \%$ [18.7\%] and 55.7\% [18.1\%], respectively; $P=0.0005)$, and this difference was mainly observed in center I $(56.9 \%$ and $62.5 \%$, respectively; $P=0.012)$ and center II ( $48.1 \%$ and $56.2 \%$, respectively; $P=0.003$ ).

DISCUSSION The present study compared knowledge about AF and anticoagulation treatment in a large patient cohort recruited from 3 Polish cardiology centers in the years 2017 and 2018. This population was larger than those previously reported. ${ }^{4,6,8}$ This report focused on regional differences with regard to the knowledge of $\mathrm{AF}$ and its management in a single country with no reimbursement system for NOAC in patients with AF. The present study shows that there are huge differences among 3 big cities with large cardiology departments, from types of oral anticoagulants used in patients with $\mathrm{AF}$, to patient knowledge on how to use these drugs safely. These observations may implicate that regional educational initiatives should be set in place for specific populations of patients with $\mathrm{AF}$ to 
improve the effectiveness and safety of anticoagulant therapy. We postulated the development and implementation of a national educational program based on validated questionnaires and directed towards patients who require lifelong anticoagulation, including those with recurrent venous thromboembolism, as they had similar knowledge gaps regarding the use of $\mathrm{OAC},{ }^{10}$ to improve clinical outcomes.

The main novel finding was that patients' knowledge was dependent on the facility type they were recruited from. Patients with AF managed in the academic reference center with a dedicated anticoagulation clinic scored significantly better than their peers from district hospitals. It might suggest that, in the era of NOAC anticoagulation, clinics pursue educational objectives directed towards patients and physicians.

Our study was conducted from 2017 to mid2018. The current real-world population was older than in most other studies, with a similar percentage of women. ${ }^{4,6,8}$ The incidence of heart failure, diabetes, and hypertension was higher than in the European Heart Rhythm Association registry, ${ }^{6}$ which confirms a common view that in everyday practice, patients with AF are "sicker" than those enrolled in selected leading centers or in randomized trials with multiple exclusion criteria. Surprisingly, we observed a very high rate of NOAC use, similar to that reported by Desteghe et al, ${ }^{4}$ but significantly higher than in other European observational studies reporting data from 2011 to $2016 .{ }^{6}$ This observation indicates that despite the fact that NOACs are quite expensive and there is no reimbursement of these agents in Poland, advantages of newer anticoagulants are important for patients and physicians, including no need for routine laboratory monitoring, fixed-dose regimens with predictable anticoagulant effects, fewer drug interactions, and no food interactions. On the other hand, our patients came from urban areas, where incomes are generally higher, and the result may be not representative for the entire Polish population. However, the highest percentage of NOAC use was in center III, which serves as a reference center for a lowincome population in Poland. This study proves that the use of NOACs is growing, even among patients with limited resources.

Unfortunately, patient general awareness of $\mathrm{AF}$ and anticoagulation treatment was insufficient, which is consistent with previous reports. ${ }^{4,6}$ As expected, older patients with more comorbidities achieved worse results. As their risk of both stroke and bleeding is higher, this observation rises concern about patient safety and indicates the urgent need for educational initiatives. As older age and the presence of multiple comorbidities are often accompanied by cognitive disorders, the educational program should also be directed towards patients' relatives and caregivers. Alarmingly, concomitant aspirin or clopidogrel use was higher than in other reports ${ }^{4,6}$ and associated with lower knowledge scores. As antiplatelet therapy combined with OAC significantly increased the bleeding risk, ${ }^{11}$ individualized targeted education about the higher bleeding risk should be proposed to this subgroup. Patients with prior myocardial infarction also gained lower scores on the JAKQ, possibly due to the fact that the attention both of the patient and physician was directed at secondary prevention of myocardial infarction, but not sufficiently focused at stroke risk associated with concomitant AF.

We observed better scores in patients with the interval from $\mathrm{AF}$ onset or the anticoagulation duration longer than 12 months. Although their associations were weak, this novel finding suggests that the longer the treatment of patients with $\mathrm{AF}$, the better their knowledge about the disease and drugs used.

In contrast to the previous study with the JAKQ in Poland, ${ }^{8}$ we found that patients on VKAs generally achieved better results than those on NOACs. A possible explanation for this observation could be a longer period between $\mathrm{AF}$ onset and OAC initiation in patients on VKAs; both factors were identified as variables positively associated with the scores in the JAKQ. It indicates that accumulating educational efforts and a larger number of ambulatory visits or hospitalizations observed in the case of several years of OAC use, improve the knowledge about $\mathrm{AF}$ and its management. It might be speculated that with time a similar phenomenon will be noted among AF patients receiving NOACs.

Our study yields insights into rapidly changing trends in anticoagulant therapy in Poland. We showed that NOACs were the preferred OACs in 2017 and mid-2018. The low prescription rate of apixaban in relation to dabigatran and rivaroxaban may be attributed to the fact that it was introduced to the Polish market in 2014 at a very high price. As real-world data indicate that apixaban use in patients with $\mathrm{AF}$ is related to lower bleeding risk, ${ }^{12,13}$ it is prescribed more often to patients with higher bleeding risk (older or on antiplatelet therapy). Of note, we found regional preferences in prescribing a specific NOAC, which are hard to explain looking at the patient characteristics, and this might be due to local preferences of the attending cardiologists. In center I, dabigatran and rivaroxaban were used similarly, while rivaroxaban was preferred in center II and dabigatran in center III. Interestingly, we observed that one-third of the patients receiving NOAC took reduced doses, which is consistent with the findings by Konieczyńska et al. ${ }^{8}$ Steinberg et $\mathrm{al}^{14}$ reported reduced NOAC doses in $16 \%$ of American patients. In the European registry, the Xarelto for Prevention of Stroke in Patients with Atrial Fibrillation (XANTUS), 
performed in 2013 and 2014, ${ }^{15}$ rivaroxaban in reduced dose was found in less than $10 \%$ of patients. The use of reduced NOAC doses in our study was associated with older age and the concomitant use of antiplatelet drugs, which indicates a higher bleeding risk perceived by physicians, despite the fact that the main indication for the NOAC dose reduction is renal insufficiency. ${ }^{1}$ It is unclear if NOAC dosing regimens were in accordance with current guidelines since creatinine clearance was not available.

The study has several limitations. It was conducted in large cardiology departments, therefore our results could not be easily translated to primary care in rural regions or in small towns. Knowledge levels of patients with AF can possibly differ between hospitals even in the same large city. We did not analyze the effect of the level of education or cognitive function of patients on AF-related knowledge. Our study was focused on the patients' knowledge about AF and its treatment and we did not collect the compliance data. Finally, it remains to be established whether the scores on the JAKQ are related to bleeding or stroke events during long-term follow-up.

In conclusion, patient knowledge on $\mathrm{AF}$ and its treatment is influenced by the type of facility they are treated at and the facility's location in the same country. Patients from a high-volume academic center achieved better scores than their peers from district hospitals. There are regional preferences in NOAC use. Further efforts are needed to improve the effectiveness of AF therapy, in part through better education with specific goals set regionally.

\section{SUPPLEMENTARY MATERIAL}

Supplementary material is available at www.mp.pl/kardiologiapolska.

\section{ARTICLE INFORMATION}

CONFLICT OF INTEREST None declared.

HOW TO CITE Janion-Sadowska A, Sadowski M, Konieczyńska M, et al. Polish regional differences in patient knowledge on atrial fibrillation and its management as well as in patterns of oral anticoagulants prescription. Kardiol Pol. 2019; 77: 437-444. doi:10.5603/KP.a2019.0036

\section{REFERENCES}

1 Kirchhof P, Benussi S, Kotecha D, et al. 2016 ESC Guidelines for the management of atrial fibrillation developed in collaboration with EACTS. Eur Heart J. 2016; 37: 2893-2962

2 Reading SR, Go AS, Fang MC, et al, Anticoagulation and Risk Factors in Atrial Fibrillation-Cardiovascular Research Network (ATRIA-CVRN) Investigators. Health literacy and awareness of atrial fibrillation. J Am Heart Assoc. 2017; 6: e005128.

3 Maccallum L, McGaw H, Meshkat N, et al. Use of an interdisciplinary, participatory design approach to develop a usable patient self-assessment tool in atrial fibrillation. Patient Prefer Adherence. 2013; 7: 1139-1146.

4 Desteghe L, Engelhard L, Raymaekers Z, et al. Knowledge gaps in patients with atrial fibrillation revealed by a new validated knowledge questionnaire. Int I Cardiol. 2016; 223: 906-914.

5 Desteghe L, Engelhard L, Vijgen J, et al. Effect of reinforced, targeted in-person education using the Jessa Atrial fibrillation Knowledge Questionnaire in patients with atrial fibrillation: a randomized controlled trial. Eur J Cardiovasc Nurs. 2019; 18: 194-203.

6 Amara W, Larsen TB, Sciaraffia E, et al. Patients' attitude and knowledge about oral anticoagulation therapy: results of a self-assessment survey in patients with atrial fibrillation conducted by the European Heart Rhythm Association. Europace. $2016 ; 18: 151-155$.

7 Steffel J, Verhamme P, Potpara TS, et al, ESC Scientific Document Group. The 2018 European Heart Rhythm Association practical guide on the use of non-vitamin $\mathrm{K}$ antagonist oral anticoagulants in patients with atrial fibrillation. Eur Heart J. 2018; 39: 1330-1393.

8 Konieczyńska M, Sobieraj E, Bryk AH, et al. Differences in knowledge among patients with atrial fibrillation receiving non-vitamin $\mathrm{K}$ antagonist oral anticoagulants and vitamin K antagonists. Kardiol Pol. 2018; 76: 1089-1096.

9 Schulman S, Angerås $U$, Bergqvist $D$, et al. Definition of major bleeding in clinical investigations of antihemostatic medicinal products in surgical patients. J Thromb Haemost. 2010; 8: 202-204.

10 Konieczyńska M, Bijak P, Desteghe L, et al. Knowledge gaps in patients with venous thromboembolism: usefulness of a new questionnaire. Pol Arch Intern Med. 2019; 129: 824-831.

11 Zalewski J, Undas A. Antithrombotic management in patients with percutaneous coronary intervention requiring oral anticoagulation. Adv Interv Cardiol. 2016; 12: 290-302.

12 Lip GYH, Keshishian A, Li X, et al. Effectiveness and safety of oral anticoagulants among nonvalvular atrial fibrillation patients. Stroke. 2018; 49: 2933-2944.

13 Bryk AH, Łukaszuk R, Donicz P, et al. Efficacy and safety of apixaban in real-life patients at high bleeding risk. Pol Arch Intern Med. 2017; 127: 889-891.

14 Steinberg BA, Shrader P, Pieper K, et al, Outcomes Registry for Better Informed Treatment of Atrial Fibrillation (ORBIT-AF) II Investigators. Frequency and outcomes of reduced dose non-vitamin $\mathrm{K}$ antagonist anticoagulants: results from ORBIT-AF II (The Outcomes Registry for Better Informed Treatment of Atrial Fibrillation II). J Am Heart Assoc. 2018; 7: e007633.

15 Pisters R, van Vugt SPG, Brouwer MA, et al. Real-life use of rivaroxaban in the Netherlands: data from the Xarelto for Prevention of Stroke in Patients with Atrial Fibrillation (XANTUS) registry. Neth Heart J. 2017; 25: 551-558. 